\title{
SAFETY DESIGN OF CAR SUSPENSION
}

\author{
Prashantha.K ${ }^{1}$, Girisha G. $\mathbf{N}^{2}$ \\ ${ }^{I}$ Electrical Engineering Department, Bangalore College of Engineering and Technology, India \\ ${ }^{2}$ Mechanical Engineering Department, Bangalore College of Engineering and Technology, India
}

\begin{abstract}
Safety mechanism is the most important factor in the automobiles. In the present study the work has been carried on safety design of car suspension. Infrared sensors were used to sense the different heights of humps on the road to protect the chassis from hitting to the humps. PLC was used to control the directional control valve. Hydraulic cylinder was used in the place of damper in the car suspension. Due to this, suspension spring do not compress fully. Now, it can able to prevent the car chassis from humps and also it resolves the customer complaint
\end{abstract}

Keywords-PLC,Cylinder,Car

\section{INTRODUCTION}

Now a day's number of new models of cars are emerging into the market. Because the customer are interested in travelling by car to have a luxury life. Some of the car manufactures are manufacturing the car in such a way that the car should go at more speeds. To achieve this more speed the ground clearance should be minimum. Because whenever the car moving at great speed at the turnings, the centrifugal force will act on the car. If the ground clearance is more then there is a chance of upsetting the car at turnings. Hence the ground clearance is minimum to avoid this upset and to meet this centrifugal force. If the ground clearance is more then the car will not go briskly at the turnings. Because of the centrifugal force acting on the car.

The construction of the humps on the road are of different types. The humps are necessary to slow down the speed at the junctions to avoid the accidents. There are different types of humps like permanent humps, temporary humps, camel shaped humps, plastic humps and bumps. Some of the humps are not effective to the car. But some are effective to the car which is having less ground clearance such as camel shaped humps.

Normally whenever the car crosses along the camel shaped humps then it tends to compress and retract back because of the load against the suspension. During this period the car tends to move up and down. Because of the impact load acting after passing on the humps the chassis is going hit to the humps. If there is only a driver then the chances of hitting to the humps is very less, because the impact load is less. The impact load is a sudden load act because of the weight of the car and then the load carrying by the car. Because of this hitting to the chassis may cause damage to the chassis and also vibrations and sounds may come gradually by frequently hitting.

Many cars having this customer complaint that chassis going to hit at the humps. The Honda civic and city is having a this customer complaint. Because of this many customers will not show interest in buying this car. Because of this the manufacturer may lose the marketing. Hence to overcome this customer complaint problem the proposed system is used.

\section{Chassis}

The Fig 1 shows the chassis and different parts under the chassis. The chassis are the skeleton of the vehicle. The chassis are the rigid body on which entire system is kept on this. The suspension is placed in between the chassis and the axle. From which the shocks from axle to chassis is not transferred, the suspension only takes the shocks.

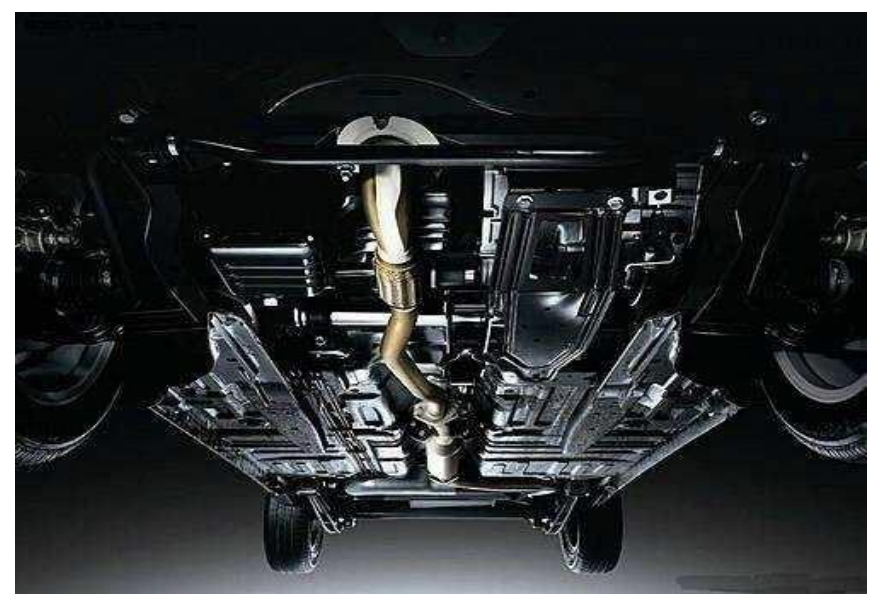

Fig 1 shows the chassis and different parts under the chassis

\section{Humps}

Humps are nothing but speed breakers. Humps are usually located in the peak areas, where pedestrians used to cross the road and at signals and at turnings or junctions. Because of these humps, vehicles will slow down and prevents accidents 


\section{Types of Humps}

Depending upon the traffic density and public, the different types of humps are constructed. Generally there are two types of humps
i. Permanent humps.
ii. Temporary humps.

\section{Permanent Humps}

Permanent humps are construct when the road is constructing by using the tar or cement. There are different shaped humps. Namely,
a) Camel shaped humps.
b) Sinusoidal humps.
c) Circular humps.
d) Parabolic humps.
e) Flat topped humps.

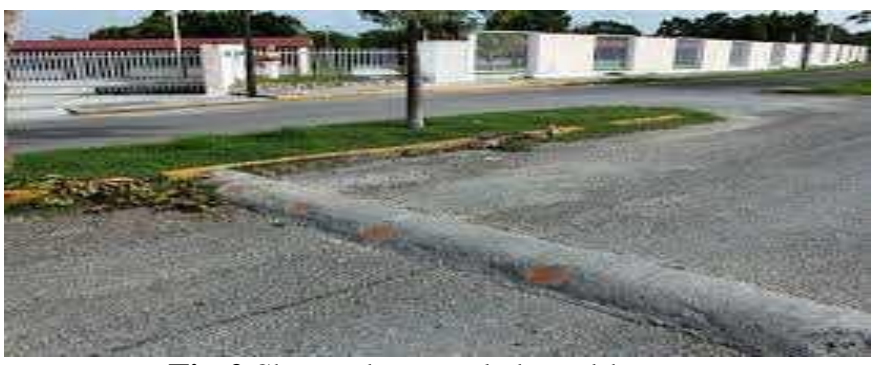

Fig 2 Shows the camel shaped humps

The Fig 2 shows that a camel shaped humps which are constructed where there is a need of maximum speed control. When there is a need to bring a vehicle into below $20 \mathrm{~km} / \mathrm{hr}$, then these types of humps are preferred. But these types of humps are harmful to the cars which are having less ground clearance. When speed is not reduces to maximum level then the chassis is going hit at that humps. These type of humps constructed near hospitals, schools, accident spots, junctions, etc. in these areas the vehicles need to go slow to avoid accidents. In these places the pedestrians used to cross the road regularly.

The Fig 3 shows that different types of humps. These types of humps constructed where the vehicles need to be slow down to some speed. When there is a need to bring vehicle into 30 to $50 \mathrm{~km} / \mathrm{hr}$, then these types of humps are preferred. These types of humps are not harmful to the cars. These humps are constructed near some signals, small junctions, carvings, etc.
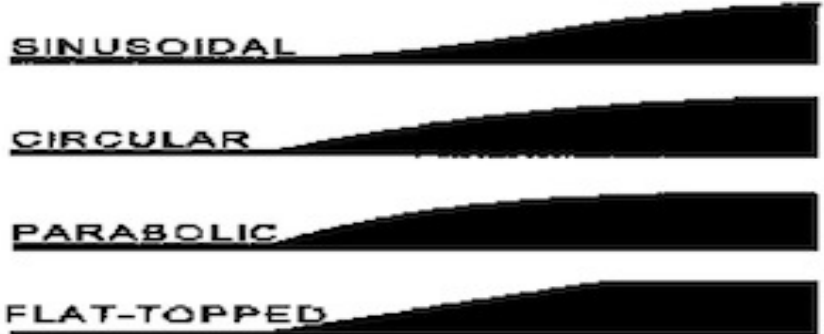

Fig 3 shows the types of humps
Temporary humpsTemporary humps are constructed by using the fibre or plastic by using bolts. These plastic humps are fixed to the road by using the bolts. This can replace or remove anytime. These are having their own standards in dimension shown in Fig4. These temporary plastics humps are going to hit back to the suspension by compression. These humps are harmful to the vehicle. Hence these are less preferable.

\section{Temporary Humps}

Temporary humps are constructed by using the fibre or plastic by using bolts. These plastic humps are fixed to the road by using the bolts. This can replace or remove anytime. These are having their own standards in dimension shown in Fig4. These temporary plastics humps are going to hit back to the suspension by compression. These humps are harmful to the vehicle. Hence these are less preferable
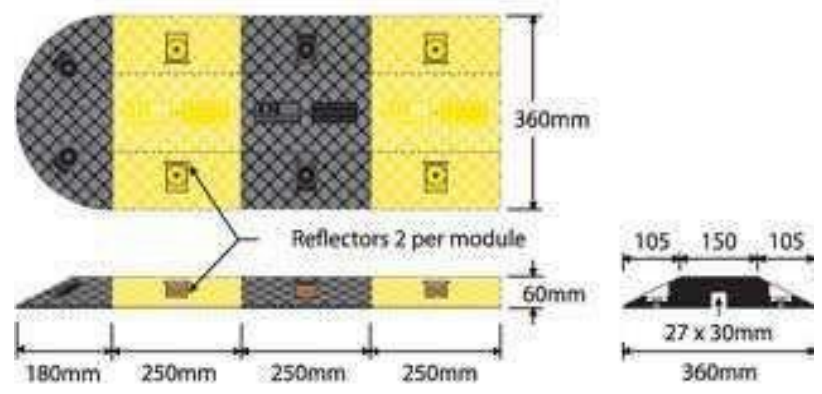

Fig 4: temporary plastic hump

\section{Suspensions}

The Fig 5 shows that a suspension with spring and damper inside the spring. Suspension is nothing but the shock absorbers. When the vehicle passes over the humps, the suspension is needed to smoothen the ride. I.e. the shock should not transmit to the passengers. Hence the suspension is placed in between the axle and the chassis of the vehicle. Hence the shock transmits from the wheel and axle is absorbed by the suspension. Which will not transmit to the chassis. Hence the passenger feels comfort. There are different types of suspension are used depending upon design of the vehicle.

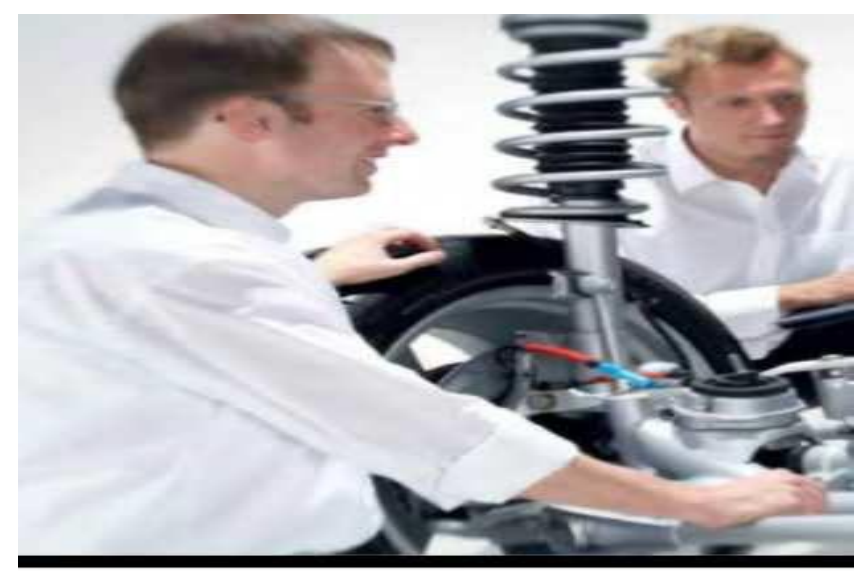

Fig 5 Shows the suspension spring with damper 


\section{PROBLEM DEFINITION}

The Fig shows the car passing along different types of humps. When the vehicle is passing over the camel shaped humps or some huge humps, there is a chances of hitting a chassis, platform and silencer depending upon the load in the vehicle, after passing the hump, the impact force will act because of the weight of the front engine and the load in the vehicle. This may cause saviour damage to the vehicle.

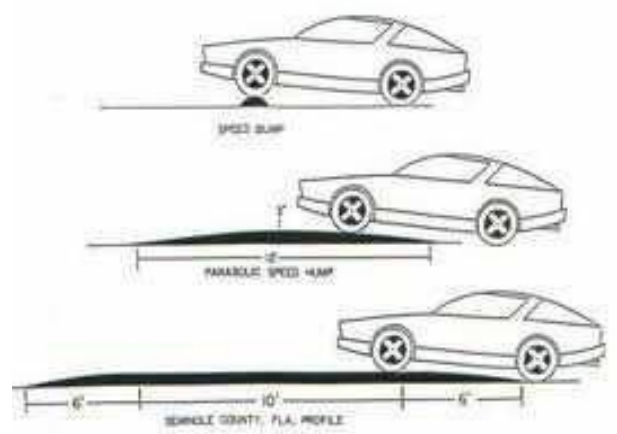

Fig 8 shows the car passing along different humps

In the view of above literature survey it has been noticed that car chassis were damagedby road hums. Hence, in the present project work an attempt has been made to design safety suspension system for car.

The hump in the road will sense by the proximity sensors which are placed at bottom front of the front bumper, and the load in the vehicle is sensed by the weighing sensor. When both signal receives, the hydraulic cylinder will lift the vehicle from the front to some certain determined measurement. By lifting this, the damage will be avoided or stopped. After passing away the humps, the back proximity sensor which is placed at bottom of the back bumper will sense the hump and gives signal to the system. Then that will come back to the normal conditions

\section{LITRATURE SURVEY}

DeoandSuh [4]has explained about the centre of gravity of a vehicle interrelated with the comfort and the handling of the vehicle. He has designed the suspension system varies with its height and stiffness according to the speed. The researcher has used a short long arm suspension system which is widely used in front wheel suspension. He controls the height

Iyad et al., [5] has introduced a new control strategy for active suspension by using fuzzy and PID controllers. By using electro hydraulic actuators he has proposed the control strategy to control the suspension system. The passive suspension is replaced by the low frequency active suspension. In this paper he has tested the car under different conditions like rolling effect, cornering and the pitching effect at different speeds and the different road profiles. By reducing the acceleration of body by $60 \%$ will gives the better road holding and car stability.

\section{DEVOLOPMENT OF PROPOSED SAFETY SUSPENSION}

\subsection{Directional Control Valve (DCV)}

The Fig 8 shows directional control valve, which is used to control the flow of the hydraulic or changing the positions. In this study, we are using 2/4 solenoid operated directional control valve shown in Fig 9. i.e. 2 positions and 4 ports namely $\mathrm{A}, \mathrm{B}, \mathrm{P} \& \mathrm{~T}$. the positions are actuated by using solenoid switch which is operated by the current.

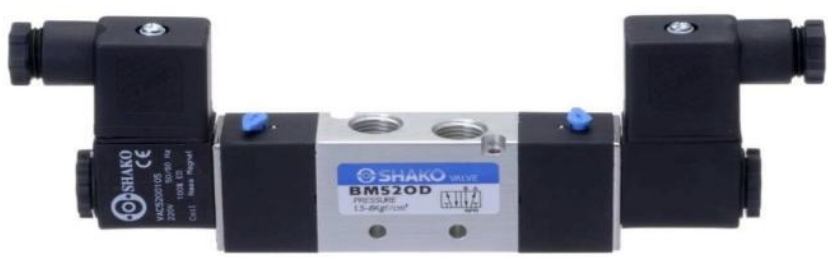

Fig 9: Direction control valve

\subsection{Hydraulic Cylinder}

The hydraulic cylinder is a actuators, which is used for the actuation of the system. There are two types of hydraulic cylinder:

- Single acting cylinders

- Double acting cylinders

\subsection{Infrared Sensors}

The infrared sensors are used to detect the object by transmitting or by detecting the infrared radiation shown in Fig 13. The infrared radiations can't be seen by the naked eyes. The infrared sensor is an electronic component which is used to sense the presence or absence of any objects in its surrounding area. In this study, the infrared sensors are used to detect the humps on the road.

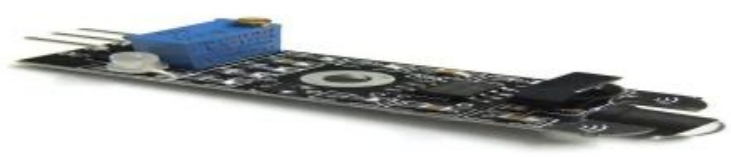

\subsection{Weighing Sensor}

Weighing sensor shown in Fig 16 is used to know the load acting on the suspension. Here we determine the actual load. If the load is under the actual load then system will not work. If the load is beyond the actual load then the IR sensors will start working

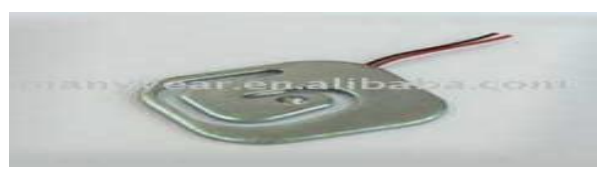

\subsection{Timer}

Timer is a electronic component which gives the timing information. Timers are used to switch on or switch off the process. The delay can be set by using of the timer 


\subsection{Process Parameters}

\subsubsection{Height of the Humps}

In this project, the sensors are used to detect the humps. This system will not work for all the humps. Only the humps which are having the more than a $12 \mathrm{~cm}$ height, such humps only sensed by the sensor.

\subsubsection{Load carrying by Car}

Normally when the load is too low, then the chassis will not hit to the humps. When load carrying is more then the specified weight, then the sensor will send the signal. Hence when ignition on the weighing sensor will sense the load carrying by the car

\subsection{Block Diagram}

The block diagram of the proposed system shown in Fig 19 consists of the load cell, 3 IR sensors, timer (T-off), PLC controller, Directional control valve and the actuator (hydraulic cylinder).

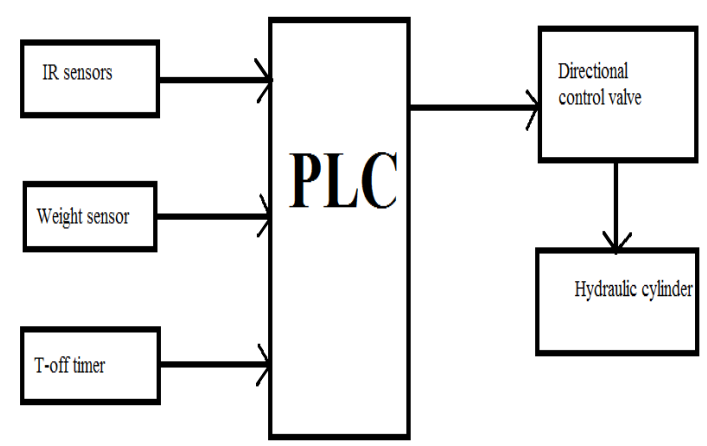

Fig 10 shows the block diagram of proposed system

\subsection{Field Setup}

The safety designs of car suspension require sensor networks. There are several methods to construct sensor networks for safety design of car suspension. The block diagram for the safety design of car suspensionis as shown in Fig 20. In the present work infrared sensors were used for sensor networks.

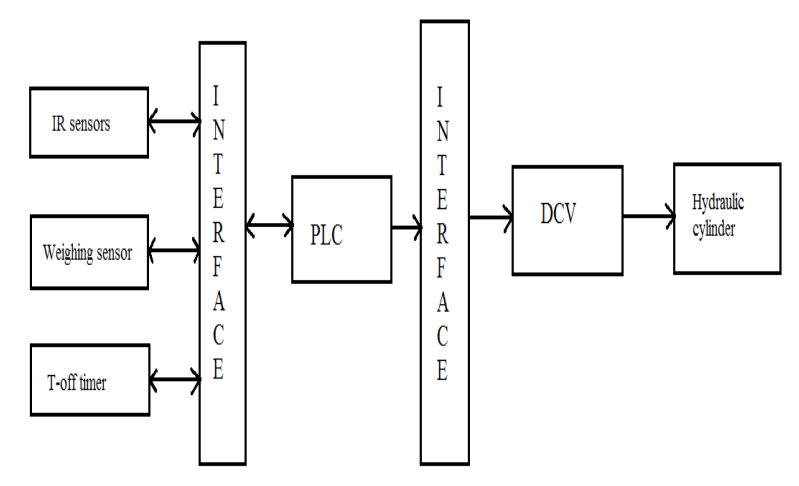

Fig 11: Proposed system field setup

\section{RESULTS AND DISCUSSIONS}

\subsection{Results}

The proposed system is discussed in the previous chapter. The final results are shown in this chapter. By implementing the proposed system into the particular car gives the following results.

\subsection{Inconventional method}

In the conventional method, the suspension system consists of wire spring with damping system. This system only avoids the sudden shocks happening on the road and protects the car. But fails in the heavy load condition.

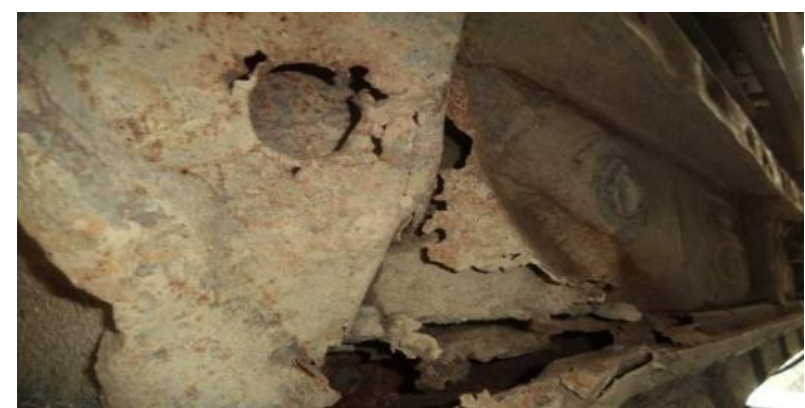

Fig 11 shows the chassis affect of hitting humps

In the above Fig 22 shows the damage of chassis on the humps due to failure of suspension system. To avoid these things, the proposed system has to be implemented

\subsection{Proposed System}

By the implementation of proposed system, the damage of the chassis is reduced. In the proposed system, the replacement of damper to the hydraulic cylinder helps to avoid the hitting of road humps to the car chassis is reduced. The implementation of proposed system to a car. In this system consists of sensor circuits and hydraulic cylinder circuits. Whenever vehicle passes over a road hump, the sensor will detect and sends signal to a controller and this will actuate the hydraulic cylinder. The actuated cylinder avoids suspension not to compress fully and thus it avoids the hitting of road hump to the car.

\subsection{Discussions}

Every customer complaint will leads to decrease its product marketing. Hence every customer complaint needs to be resolve to satisfy the customers and making him to buy the product. The chassis hitting to the humps on the road when it is fully loaded is also the customer complaint in some sedan cars. The sedan cars are made to go faster in road and also the enough space for the customers. To achieve the maximum speed, the car will design to meet its speed.

The ground clearance of the car is the important factor should be considered while designing for its speed. Whenever the car is moving on the turning roads, then the car leads to attract outside because of the centrifugal force acting on the car outside to the curving of the road. 
When the ground clearance is more, then the car leads to upset by its weight and centrifugal force. Hence the designer used to keep the ground clearance less as possible. Because of this less ground clearance, the car weight used to act more towards down and also it holds the road and it will not allow car to upset by its centrifugal force. Because of this less ground clearance the chassis of the car will used strike the hump on the road, whenever the car is fully loaded.

The humps are used to slow down the speed of the car. There are different types of humps depending upon the required slowdown of the car. The flat topped and circular humps are used to slowdown the car to its minimum decrease. The camel shaped humps are used to decrease the speed of the car to its maximum decrease.

In such camel shaped humps, the car used to slowdown but when it is fully loaded then the car chassis is used to strike on the humps. The suspension will compress more against the spring. Hence it is going to strike on the humps. This is the customer complaints for some sedan cars. Due to this reason only, it has loosed its sale.

To overcome this problem, the proposed system was used. In proposed system, the damper was replaced by the hydraulic cylinder with the spring on one side of the piston. Here the hydraulic cylinder should act as a damper in normal condition. In which it is used to absorb the shocks produced by the spring and the uneven surface on the road. The suspension is used to absorb these shocks by preventing by sending these shocks to the body of the car. When the humps are sensed, then the hydraulic cylinder will raise to avoid the stroke of the chassis to the car.

The infrared sensors are used to detect the humps on the road. The IR sensors are set to some distance depending upon the ground clearance of the car. The humps which are more than $12 \mathrm{~cm}$ height will detect by sensors. The IR sensors are placed in front bottom of the front bumper of the car. The sensor will send the pulse signal; to the PLC, whenever the humps are detected. This is latched because, this input pulse signal should give a output for some time. Hence, circuit needs to be latched.

The timer is used to switch off the circuit. The T-off timers are used to switch off the circuit after 4 secs. When IR sensors detect the hump, then PLC will send signal to the directional control valve. Which causes the hydraulic cylinder to lift. After $4 \mathrm{sec}$, the T-off timer will switch off the circuit and brings back to the normal condition until the next hump is detect by car.

The directional control valve is used to control the movement of the cylinder. In the proposed system, the $4 / 2$ single solenoid, spring return valve is used. 4 ports and 2 positions are used. It is actuated by solenoid by using current. When the current is off in the solenoid then the spring will bring back to its normal position by spring return.

\section{CONCLUSION AND SCOPE OF FUTURE WORK}

\subsection{Conclusion}

- The aim of this project was to avoid the damages by hitting of chassis at the humps and it has been avoided.

- The damper in the suspension is replaced by hydraulic cylinder.

- IR sensors were used actively detect the humps which are more than $12 \mathrm{~cm}$ in height

- The PLC was used dynamically controls the proposed safety suspension system

- $\quad$ The customer complaints was satisfied

\subsection{Future Scope}

- The PLC can be replaced by Microprocessor

- The input from the weighing sensor can be used for any other application.

- The hydraulic system can be used for some other applications like for jack lifting, steering unit, etc.

\section{REFERENCES}

[1]. Donald. L. Anglin, Automobile Engineering.

[2]. Heller, Carl T., Automotive Braking Systems (Reston Publishing Company Inc., 1985).

[3]. Stroll \&Bernaud, Hydraulic Control System, Tata McGraw Hill Publications.

[4]. Hrishikesh V Deo, Nam P Suh -Axiomatic Design Of Customizable Automotive Suspension

\section{BIOGRAPHIES}

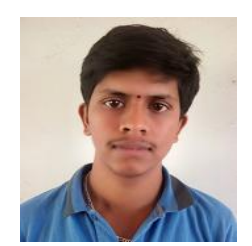

Girisha $\mathbf{G} \quad \mathbf{N}$ Studying 8th sem B.E Mechanical engineering from Bangalore college of engineering and technology

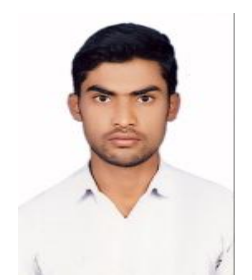

Prashantha $\mathbf{k}$ is currently Asst prof, in the Department of Electrical and electronics engineering, BCET,bangalore Received M. Tech. degree in Industrial drives and automation engineering From VTU-RC Mysore And B.E Electrical and electronics engineering from Sri Sairam Engineering, Bangalore (VTU) in 2013. 